\title{
COMPARACIÓN DE DOS MÉTODOS CONVENCIONALES DE DIAGNÓSTICO DE NEMATODES INTESTINALES EN Canis familiaris CON EL EXAMEN POST-MORTEM
}

\author{
José Díaz P. ${ }^{1}$, Amanda Chávez V. ${ }^{2}$ y Eva Casas A. ${ }^{2}$
}

\section{Abstret}

The diagnostic effectiveness of two parasitological tests in feces, the simple direct method and the flotation method, were compared against post-mortem findings utilizing samples collected from 180 street dogs euthanized during a rabies control campaign. Post-mortem examination revealed that $27 \%$ of the animals were infected with tapeworm (Toxocara canis and Toxascaris leonina), but the simple direct method and the flotation method only indicated $12.7 \%(0.47+0.07$ sensibility) and $17.2 \%(0.63+0.07$ sensibility $)$, respectively. Kappa and McNemar analysis showed statistical differences and an agreement index of $0.68 \pm 0.07$ between both tests. The results produced by the simple direct and flotation methods were weakly and moderately proportional to the post-mortem findings, indicating that the flotation method is more efficient than the simple direct method, although both methods may produce negative results for cases with a low level of parasitism.

Key words: Fecal tests for endoparasites, dogs, Toxocara.

\section{Resumen}

Se comparó la efectividad diagnóstica de dos exámenes coprológicos, el método directo simple y el de flotación con solución saturada de cloruro de sodio, con los resultados obtenidos al examen post-mortem. Muestras de heces de 180 canes sacrificados por el Centro Antirrábico de Lima fueron recolectadas para determinar la carga parasitaria. Al examen post-mortem se encontró que el $27 \%$ de los animales presentaban infección por ascaroideos (Toxocara canis y Toxascaris leonina). Sin embargo, los métodos directo simple y de flotación sólo alcanzaron a diagnosticar como muestras positivas a un 12.7 y $17.2 \%$. Se determino índices de sensibilidad de $0.47 \pm 0.07$ para el método directo simple y de $0.63 \pm 0.07$ para el método de flotación. Con los análisis de Kappa y Mc Nemar se obtuvo un índice de concordancia de $0.68 \pm 0.07$ con diferencias estadísticas significativas entre los resultados de ambas pruebas. Se concluye que el método de flotación es más eficiente como prueba diagnóstica que el método directo simple, aunque hay que tener en consideración que los resultados de ambas pruebas tenderán a ser negativos en infecciones leves.

Palabras clave: Diagnóstico coprológico, perro, Toxócara.

1 Práctica privada

2 Laboratorio de Parasitología - FMV - UNMSM. E.mail:. A_CHAVEZ_G@HOTMAIL.COM 


\section{Introitrecion}

El parasitismo intestinal constituye uno de los principales problemas en la sanidad de caninos, sobre todo en cachorros, por el desmedro que producen en su salud y condición general, pudiendo causar en casos masivos, la muerte de camadas enteras (Soulsby, 1987). Los cachorros son frecuentemente infectados por parásitos ascaroideos, ya sea a través de las vías transplacentaria y lactogénica o por ingestión directa de huevos infectantes, ocasionando infección intestinal que se manifiesta en las primeras semanas de vida. Los perros parasitados pueden llegar a contaminar el medio ambiente con enormes cantidades de huevos y larvas infectivas, constituyendo así un serio peligro para la salud pública. Es por ello importante detectar a tiempo la presencia de estos parásitos, para lo cual existen diferentes pruebas de diagnóstico.

Dentro de las pruebas parasitológicas de rutina utilizadas para el diagnóstico del parasitismo intestinal en caninos, se tienen las pruebas cualitativas, que en la mayoría de los laboratorios de parasitología se utilizan con frecuencia para el diagnóstico de helmintos intestinales. Dentro de éstas, el método directo simple y el método de flotación son los más usados.

El objetivo del presente trabajo fue comparar los resultados del diagnóstico de nematodes intestinales, obtenidos por los métodos directo simple y de flotación con solución de cloruro de sodio, con el recuento total de parásitos obtenidos al examen postmortem, así como determinar la prevalencia de nemátodes intestinales en caninos sacrificados en el Centro Antirrábico.

\section{Materiales y Mrobius}

Se colectaron muestras de heces y parásitos intestinales de 180 perros vagos y hogareños, procedentes de diferentes sectores de Lima metropolitana, que fueron colec- tados y sacrificados por el Centro Antirrábico de Lima de acuerdo al plan establecido de control de rabia. Las muestras fueron trasladadas al laboratorio de Parasitología de la Facultad de Medicina Veterinaria de la UNMSM, para los análisis coprológicos respectivos.

Se realizó el método directo simple (MDS), según la técnica descrita por Weybridge laboratories (1973) y el método de flotación (MF) con solución de Cloruro de Sodio $(\mathrm{ClNa})$, según el procedimiento descrito por Melvin y Brooke (1971). La identificación de nemátodes se realizó con la ayuda de un estereoscopio observando las características morfológicas descritas por Soulsby (1987) y Leguía (1996).

La sensibilidad de las pruebas se determinó confrontando sus resultados con los obtenidos mediante el examen post-mortem. Se utilizó la prueba de Kappa y McNemar para determinar el nivel de concordancia. Adicionalmente se investigó la correlación entre las cargas parasitarias a la necropsia versus los resultados de los métodos directo y de flotación.

\section{Resulbuins}

El Cuadro 1 muestra los resultados de la evaluación post-mortem de 180 perros, donde se encontró que el $27.2 \%$ de ellos se encontraban parasitados por ascaroideos, correspondiendo el $13.9 \%$ a infecciones por Toxocara canis y el $13.3 \%$ a Toxascaris leonina. El nivel de infección parasitaria, clasificado a través de infecciones leves (1-2 parásitos), moderadas (3-10 parásitos) y masivas ( $>10$ parásitos) fue relativamente simi$\operatorname{lar}(19,13$ y 17 casos respectivamente).

En el Cuadro 2 se comparan los resultados de las dos pruebas de diagnóstico con el examen post-mortem. La efectividad del MDS fue del $46.9 \%$ en tanto que del MF fue del $63.3 \%$. muestras positivas, respectivamente de un total de 49 animales infecta- 
Cuadro 1. Frecuencia y nivel de infección de Toxocara canis y Toxascaris leonina en caninos mediante el examen post-mortem, Lima, 1999.

\begin{tabular}{lccccc}
\hline \multirow{2}{*}{ Especie } & \multicolumn{3}{c}{ Nivel de Infección } & \multirow{2}{*}{$\begin{array}{c}\text { Frecuencia de } \\
\text { Infección Parasitaria }\end{array}$} \\
\cline { 2 - 6 } & Negativo & Leve & Moderado & Masivo & $(\%)$ \\
\hline T. canis & 155 & 12 & 05 & 08 & 13.89 \\
T. leonina & 156 & 07 & 08 & 09 & 13.33 \\
\hline \multicolumn{1}{c}{ Total } & 131 & 19 & 13 & 17 & 27.22 \\
\hline Negativo : 0 parásitos & & & & \\
Leve : 1-2 parásitos & & & & \\
Moderado: $:-10$ parásitos & & & & \\
Masivo : Más de 10 parásitos & & & & &
\end{tabular}

Cuadro 2. Comparación de la efectividad de las pruebas de diagnóstico nematológico con el examen post-mortem, Lima, 1999.

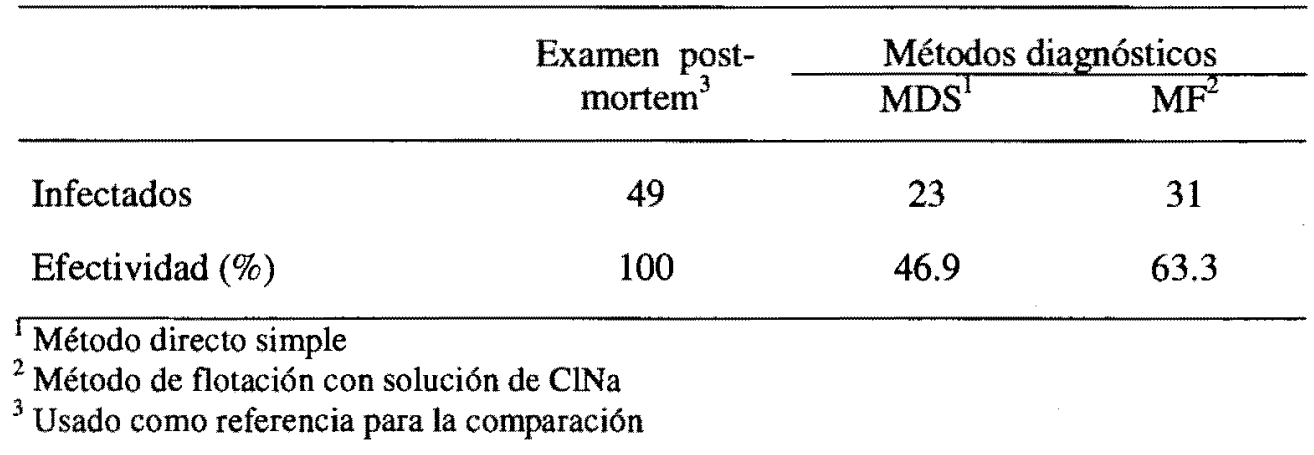

dos por ascaroideos. El MDS detectó 16.3\% casos positivos menos que el MF.

La Fig. 1 muestra la comparación de la efectividad de los métodos diagnósticos en estudio, según sea el nivel de infección parasitario leve, moderado o masivo. El MF tuvo una mayor efectividad que el MDS en todos los niveles de infección (leve: $42.1 \pm 11.3 \mathrm{vs}$ $21.0 \pm 9.5 \%$; moderado: $53.9 \pm 13.8$ vs 46.2 $\pm 13.8 \%$; masiva: $94.1 \pm 5.7$ vs $76.5 \pm$ $10.3 \%)$.

La sensibilidad hallada para el MDS y MF fue de $47 \pm 7$ y $63 \pm 7 \%$, respectivamente. Los análisis Kappa y Mc Nemar demostraron que ambos métodos presentan un índice moderado de concordancia $(0.68 \pm 0.07$
$\%)$. Sin embargo existen diferencias estadísticas significativas en la efectividad. Además se determinó que las cargas parasitarias obtenidas a la necropsia y los resultados de las pruebas evaluadas muestran una correlación directamente proporcional, siendo en grado débil para el MDS y en grado moderado para el MF.

\section{Discusión}

Acha (1952) y Triveño (1970) encontraron prevalencias de 21 y $10 \%$ para Toxocara canis y de 29 y $34 \%$ para Toxascaris leonina en estudios realizados en perros de la ciudad de Lima. En el presente estudio, las tasas de frecuencia encon- 


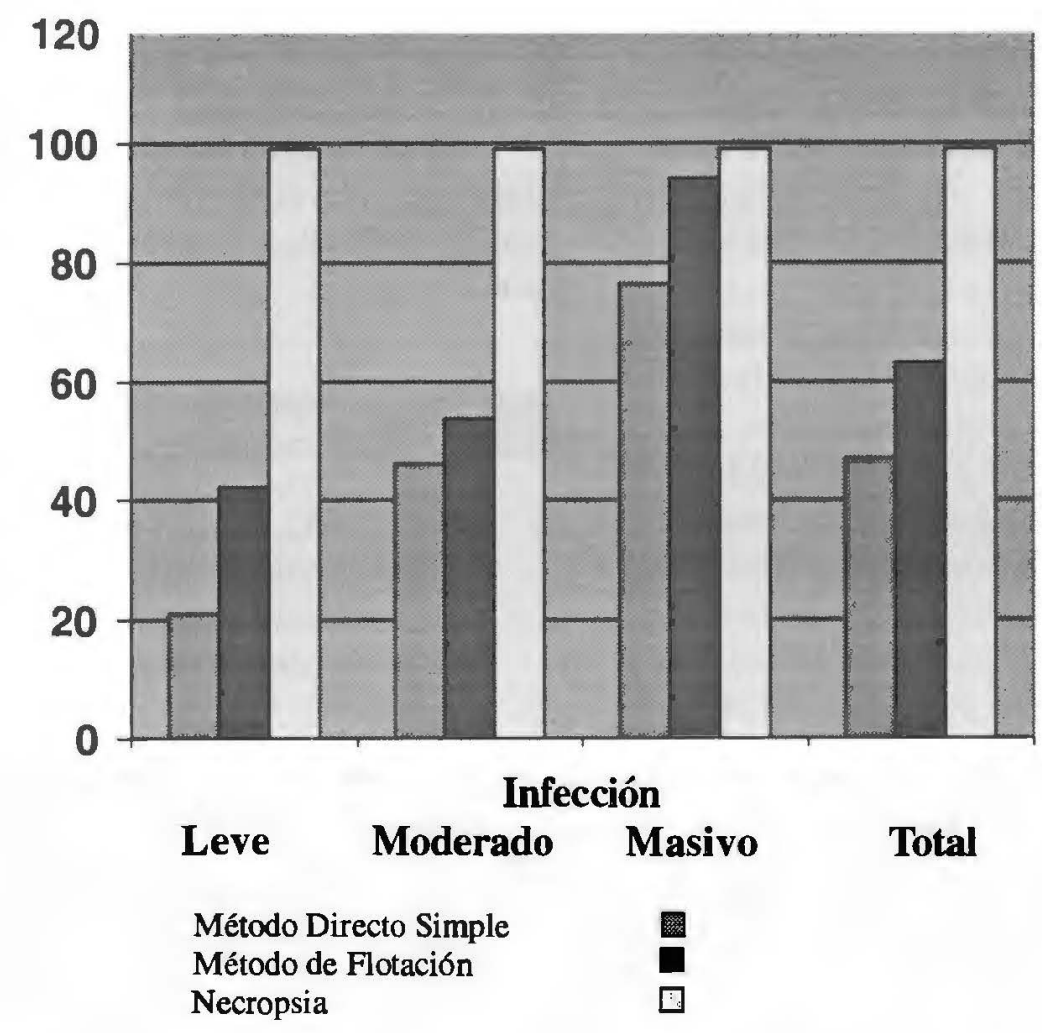

Figura 1. Comparación de la efectividad de las pruebas de diagnóstico nematológico con el examen post-mortem, según el nivel de infección parasitaria.

tradas (13.9\% para $T$. canis y $13.3 \%$ para $T$. leonina) indican una reducción apreciable de las prevalencias obtenidas anteriormente, lo cual podría explicarse por las medidas de control antiparasitario que en la actualidad se practican cada vez con mayor frecuencia. Por otro lado Georgi y Georgi (1994) manifiestan que las infecciones por T. leonina no son tan comunes como las producidas por $T$. canis debido a la carencia de invasividad de sus larvas a los tejidos caninos y humanos, impidiendo la transmisión vertical de la infección.

En la práctica rutinaria de la mayoría de consultorios veterinarios, se utiliza el método directo simple con una muestra obtenida luego de colocar el termómetro en el recto del animal, cuando se desea realizar un examen parasitologico en forma rápida. Sólo los consultorios que cuentan con facilidades de laboratorio realizan alguna técnica de concentración para obtener un diagnóstico más confiable.

En el presente estudio se determinó que menos del $50 \%$ de los casos positivos fueron detectados por el método directo simple, mientras que con el método de flotación con CINa se logró detectar un $63 \%$ de ellos. Los resultados, con el primer método se alejan de un diagnóstico adecuado y preciso, pues no logró demostrar la presencia de huevos en las heces de 26 animales infectados. Sin embargo, no se puede condenar a este método pues hay que considerar que 19 de las muestras pertenecían a individuos con infecciones leves (1-2 parásitos), y según la literatura, este método detecta mayormente infecciones masivas (Leguía, 1996) o por lo menos corı una elevada concentración de huevos (Weybridge laboratories, 1973). Por lo tanto, el método directo simple se puede considerar como una prueba de baja efectividad en infecciones leves y moderadas. 
Según Weybridge laboratories (1973) y Leguía (1996), el método de flotación utilizando una solución saturada de ClNa es el más indicado para el diagnóstico de ascaroideos, pudiendo detectar incluso infecciones leves. No obstante, si bien este método tiende a ser más efectivo que el método directo simple, no otorga plena garantía en su utilización pues no logró diagnosticar a 18 animales positivos. Los mejores resultados de diagnóstico fueron en perros con infecciones masivas (>10 parásitos), alcanzando un $94 \%$ de eficiencia, pero contrastando con lo obtenido en infecciones leves (1-2 parásitos) donde la eficiencia estuvo por debajo del $50 \%$.

Los índices de sensibilidad de $47 \%$ y $63 \%$, obtenidos para el método directo simple y el método de flotación con $\mathrm{ClNa}$, respectivamente, confirman la eficiencia relativa que demuestran estos métodos en el diagnóstico de nemátodes, comparados con el examen de necropsia. La baja efectividad de los métodos directo simple y de flotación puede atribuirse a diferentes factores, tales como la distribución desuniforme de los huevos, la intermitencia en la puesta de huevos parasitismo de un sólo de sexo y la resistencia del hospedador (Weybridge laboratories, 1973).

\section{Conclusiones}

El método de flotación con solución saturada de $\mathrm{ClNa}$ es más eficiente que el método directo simple. Sin embargo, por sus bajos índices de sensibilidad en infecciones leves y moderadas, un resultado negativo de cualquiera de estas pruebas no garantiza la ausencia de nemátodes intestinales.

El diagnóstico positivo que se obtenga al utilizarse el método directo simple puede considerarse indicativo de una infección masitủile nemátodes intestinales.

\section{Bthliografía}

1. Acha, P. 1952. Porcentaje de Parasitosis del Canis familiaris en la ciudad de Lima. Tesis de Bachiller. Fac. Med. Vet. Univ. Nac. Mayor de San Marcos, Lima. Perú. p.18-26.

2. Georgi, J. y M. Georgi. 1994. Parasitología en Clínica Canina. p.174179. Interamericana. México.

3. Leguía, G. 1996. Enfermedades Parasitarias de Perros y Gatos. Epidemiología y Control. p.18, 19, 25. Ed. del Mar EIRL. Lima-Perú.

4. Melvin, D. y M. Brooke. 1971. Métodos de laboratorio para diagnóstico de parásitos intestinales. Interamericana. México. p. 198.

5. Soulsby, E. 1987. Parasitología y enfermedades parasitarias en los animales domésticos. $7^{\mathrm{ma}}$ ed. p.150-152. Nueva editorial Interamericana. México.

6. Triveño, F. 1970. Estudio del Diphyllobotrium y otros Helmintos en Humanos, gatos, perros y peces de la ciudad de Lima. Tesis de Bachiller Fac. Med. Vet. Univ. Nac. Mayor de San Marcos, Lima. Perú. p.21-23.

7. Weybridge Laboratories. 1973. Manual de Técnicas de Parasitología Veterinaria. p.10-19, 59, 60. Ed. Acribia, Inglaterra. 